\title{
DỨBin
}

Technological University Dublin ARROW@TU Dublin

\section{Bismuth-Doped Glass Microsphere Lasers}

\section{Zaijn Fang}

Harbin Engineering University, China

Sile Nic Chormaic

2Light-Matter Interactions Unit, Okinawa Institute of Science and Technology Graduate University, Okinawa, Japan

Shanyu Wang

1 Key Laboratory of In-Fiber Integrated Optics, Ministry Education of China, Harbin Engineering University, Harbin 150001, China

See next page for additional authors

Follow this and additional works at: https://arrow.tudublin.ie/engscheceart

Part of the Engineering Commons

\section{Recommended Citation}

Wang, P., NicChormaic, S. \& Wang, S. (2017). Bismuth-doped glass microsphere lasers. Photonics Research, vol. 5, no. 6, pp. 740-744. doi.org/10.1364/PRJ.5.000740

This Article is brought to you for free and open access by the School of Electrical and Electronic Engineering at ARROW@TU Dublin. It has been accepted for inclusion in Articles by an authorized administrator of ARROW@TU Dublin. For more information, please contact arrow.admin@tudublin.ie, aisling.coyne@tudublin.ie, gerard.connolly@tudublin.ie.

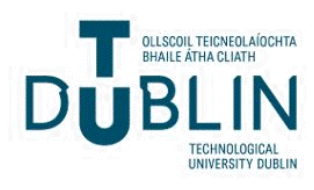




\section{Authors}

Zaijn Fang, Sile Nic Chormaic, Shanyu Wang, Xin Wang, Jibo Yu, Yuxuan Jiang, Jianrong Qiu, and Pengfei Wang

This article is available at ARROW@TU Dublin: https://arrow.tudublin.ie/engscheceart/170 


\title{
Bismuth-doped glass microsphere lasers
}

\author{
Zaijin Fang, ${ }^{1}$ Síle Nic Chormaic, ${ }^{2}$ Shanyu Wang, ${ }^{1}$ Xin Wang, ${ }^{1}$ Jibo Yu, ${ }^{1}$ Yuxuan Jiang, ${ }^{1}$ \\ Jianrong Qiu, ${ }^{3,5}$ and Pengfei Wang ${ }^{1,4, *}$ \\ ${ }^{1}$ Key Laboratory of In-Fiber Integrated Optics, Ministry Education of China, Harbin Engineering University, Harbin 150001, China \\ ${ }^{2}$ Light-Matter Interactions Unit, Okinawa Institute of Science and Technology Graduate University, Onna, Okinawa 904-0495, Japan \\ ${ }^{3}$ State Key Laboratory of Modern Optical Instrumentation, Zhejiang University, Hangzhou 310027, China \\ ${ }^{4}$ Photonics Research Centre, Dublin Institute of Technology, Kevin Street, Dublin 8, Ireland \\ ${ }^{5}$ e-mail: qjr@zju.edu.cn \\ ${ }^{*}$ Corresponding author: pengfei.wang@dit.ie
}

Received 13 July 2017; revised 10 October 2017; accepted 26 October 2017; posted 27 October 2017 (Doc. ID 302404); published 22 November 2017

\begin{abstract}
In this work, a hybrid structure consisting of a multicomponent germanate glass microsphere containing bismuth as a gain medium is proposed and presented. The bismuth-doped germanate glass microspheres were fabricated from a glass fiber tip with no precipitation of the bismuth metal. Coupling with a fiber taper, the bismuth-doped microsphere single-mode laser was observed to lase at around $1305.8 \mathrm{~nm}$ using $808 \mathrm{~nm}$ excitation. The low threshold of absorbed pump power at $215 \mu \mathrm{W}$ makes this microlaser appealing for various applications, including tunable lasers for a range of purposes in telecommunication, biomedical, and optical information processing. (๑) 2017 Chinese Laser Press
\end{abstract}

OCIS codes: (140.3380) Laser materials; (140.3945) Microcavities; (160.2750) Glass and other amorphous materials.

https://doi.org/10.1364/PRJ.5.000740

\section{INTRODUCTION}

In recent decades, gain glass materials with ultrabroadband emission have been intensely investigated due to their various invaluable applications, such as broadband fiber amplifiers and tunable fiber lasers $[1,2]$. Nevertheless, most investigations in relation to luminescent glasses are confined to materials with dopants of a range of rare-earth ions, such as $\mathrm{Yb}^{3+}, \mathrm{Er}^{3+}$, $\mathrm{Tm}^{3+}$, and $\mathrm{Ho}^{3+}$. Owing to the shielding of the $6 s$ and $5 d$ orbitals, the emission wavelength is fixed, and the bandwidth can be narrow $(<100 \mathrm{~nm})$ due to the $4 f-4 f$ orbital transition nature of rare-earth ions, preventing wide applications of luminescent glasses at unconventional wavelengths [3-5]. Bismuth (Bi)-doped glasses exhibit ultrabroadband lasing emissions in the near-infrared (NIR) region ranging from 1000 to $1700 \mathrm{~nm}$, and the FWHM of the luminescence can range from $200 \mathrm{~nm}$ to nearly $500 \mathrm{~nm}[6-14]$. Furthermore, the NIR emission spectra of Bi-doped glasses (around $1310 \mathrm{~nm}$ ) effectively fill up the vacant spectral region $(1100-1400 \mathrm{~nm})$ of traditional rare-earth-doped active glasses. These unique luminescent properties indicate that $\mathrm{Bi}$-doped glasses are good candidates for an ultrabroadband lasing application at unconventional wavelengths and for tunable lasers. Previously, a novel fiber laser material based on Bi-doped silica glass emitting in the NIR region garnered a lot of attention [15-22]. However, the doping concentrations of $\mathrm{Bi}$ in silica glass are low (normally less than $0.1 \mathrm{~mol} \%$ ), the preform is fabricated at a high temperature (about $2000^{\circ} \mathrm{C}$ ) with heavy volatility of $\mathrm{Bi}$, and the emission bandwidths of the fibers are narrower than that of Bi-doped bulk glasses. In comparison with silica glass, the doping concentrations of $\mathrm{Bi}$ in multicomponent glasses can be much higher, and the melting temperatures are much lower. In addition, the gain coefficients of $\mathrm{Bi}$ in multicomponent glasses are higher than in silica glass due to their low multiphonon relaxation rates. Therefore, Bi-doped multicomponent glass is a promising matrix for lasing applications. As far as the authors are aware, no investigation into Bi-doped multicomponent glass fiber lasers has been reported in the literature due to the high transmission loss of fibers and high pump threshold of Bi-doped multicomponent fiber laser.

Whispering gallery modes (WGMs) in glass microcavities exhibit unique properties of extremely high quality factors (up to $10^{10}$ ) and very small mode volumes (on the order of $100 \mu \mathrm{m}^{3}$ ) [23,24]. The strong confinement of light makes microsphere resonators attractive as resonant cavities for laser oscillation, especially for low threshold and high pump efficiency lasers $[25,26]$. Therefore, microsphere resonators should also be promising for Bi-doped multicomponent glass lasers due to the unique property of the ultrahigh electromagnetic energy density. In this work, Bi-doped multicomponent germanate glass microspheres were prepared, and a Bi-doped microsphere laser in the NIR region was demonstrated. The Bi-doped multicomponent glasses were characterized using 
both transmission spectra and X-ray diffraction $(\mathrm{XRD})$ patterns. The fluorescence luminescence of the Bi-doped glass was measured over an ultrabroadband of $1000-1700 \mathrm{~nm}$ wavelength range under $808 \mathrm{~nm}$ laser excitation. The microspheres were fabricated from glass fibers, which were drawn from melted Bi-doped germanate glass. A Bi-doped microsphere laser was generated and observed to operate in the NIR region around $1310 \mathrm{~nm}$ using the $808 \mathrm{~nm}$ excitation.

\section{EXPERIMENTS}

To fabricate the microspheres, we used a Bi-doped germanate glass with the compositions (mol\%): $70 \mathrm{GeO}_{2}-15 \mathrm{Ga}_{2} \mathrm{O}_{3}$ $(15-x) \mathrm{BaO}-x \mathrm{Bi}_{2} \mathrm{O}_{3}(x=1.0,2.0,3.0,3.5$, and 4.0). A $30 \mathrm{~g}$ reagent grade stoichiometric mixture of $\mathrm{GeO}_{2}$ (99.999\%), $\mathrm{Ga}_{2} \mathrm{O}_{3}$ (99.999\%), $\mathrm{BaCO}_{3}$ (99.99\%), and $\mathrm{Bi}_{2} \mathrm{O}_{3}$ (99.999\%) was mixed thoroughly in an agate mortar and melted in a covered corundum crucible at $1350^{\circ} \mathrm{C}$ for $1 \mathrm{~h}$. The bulk glass samples were fabricated using the melting-quenching method. The glass samples were cut and polished for measurements. Then, the germanate glass fibers were drawn from the glass melt using a diamond tip, similar to that described elsewhere [27]. The glass fibers were prepared by quickly drawing at an appropriate glass melt viscosity, which was initially cleaned ultrasonically and then using a number of hydrofluoric acid washes.

To fabricate the Bi-doped germanate microsphere, a taper from the glass fiber was first prepared by vertically suspending it on a $3 \mathrm{D}$ adjustable mount. A small region of the fiber was heated by a $\mathrm{CO}_{2}$ laser beam focused to about $150 \mu \mathrm{m}$, and we increased the power of the laser until the glass fiber rapidly elongated into a taper. We were able to control the waist diameter of the taper by adjusting the distance between the glass fiber and the laser beam; a taper diameter of about $3 \mu \mathrm{m}$ was selected, and it was then cut into a half-taper by shining the laser at the center of the waist region. Next, we heated the tip of the half-taper by the $\mathrm{CO}_{2}$ laser beam until it was near the melting temperature of the glass. The surface tension of the glass creates a microsphere from the tip. During the heating process, the tapered fiber was moved slowly along the half-taper in order to enlarge the size of the glass microsphere. Using the described method, we made Bi-doped germanate microspheres with diameters ranging from 40 to $100 \mu \mathrm{m}$. Finally, the Bi-doped germanate microspheres were used as gain matrices as well as resonant cavities to realize Bi lasers.

For characterizing the absorption properties of the doped germanate glasses, the transmission spectra were measured using an ultraviolet-visible-NIR spectrophotometer (Lambda-900, PerkinElmer, USA). The amorphous state and crystalline phase in the glasses, glass fibers, and microspheres were identified by XRD patterns on a D8 advance x-ray diffractometer (Bruker, Switzerland) with $\mathrm{Cu} / \mathrm{Ka}(\lambda=0.1541 \mathrm{~nm})$ radiation. The photoluminescence spectra of the Bi-doped germanate glasses were measured using an FLS920 fluorescence spectrometer (Edinburgh Instruments, UK). Lasing from the Bi-doped microspheres was detected using an optical spectrum analyzer (AQ-6370C, Yokogawa, Japan).

\section{EXPERIMENTAL RESULTS AND DISCUSSION}

Figure 1(a) shows the measured transmission spectra for Bi-doped germanate glasses as a function of wavelength for different doping concentrations. Two characteristic absorption bands around 500 and $800 \mathrm{~nm}$ are observed (see highlighted regions); these are attributed to electronic transitions of $\mathrm{Bi}$, as previously reported $[28,29]$. The absorption intensity of $\mathrm{Bi}$ increases monotonically as the doping concentration of $\mathrm{Bi}_{2} \mathrm{O}_{3}$ increases. In the 1000-2000 nm region, the transmittance of the glasses is as high as $80 \%$ when the thicknesses of the glasses are $1.5 \mathrm{~mm}$.

According to the absorption properties of $\mathrm{Bi}$ in the glasses, as described above, the photoluminescence spectra were measured under excitation of an $808 \mathrm{~nm}$ laser diode. The dependence of the NIR emission intensity on the doping concentration of $\mathrm{Bi}_{2} \mathrm{O}_{3}$ is presented in Fig. 1(b). On excitation by $808 \mathrm{~nm}$ light, ultrabroadband NIR emissions are observed in the photoluminescence spectra. The center of the emission bands is located at $1280 \mathrm{~nm}$, and the FWHM of the emissions is about $270 \mathrm{~nm}$. The ultrabroadband emissions have been attributed to electronic transitions of low valence $\mathrm{Bi}^{+}$or $\mathrm{Bi}$ clusters [30-33], but the exact origin would need further investigation. Furthermore, when excited at $808 \mathrm{~nm}$, the ultrabroadband emission intensity of $\mathrm{Bi}$ first increases as the doping concentration of $\mathrm{Bi}_{2} \mathrm{O}_{3}$ is increased, reaching a maximum at $3.5 \mathrm{~mol} \%$ $\mathrm{Bi}_{2} \mathrm{O}_{3}$ doping, and then decreases when the $\mathrm{Bi}_{2} \mathrm{O}_{3}$ content is further increased. This result can be understood by considering the effect of concentration quenching of Bi due to the energy migration between $\mathrm{Bi}$ ions. The optimal doping concentration of $\mathrm{Bi}_{2} \mathrm{O}_{3}$ for NIR emission in this glass is approximately $3.5 \mathrm{~mol} \%$.

In terms of the experimental results mentioned above, $3.5 \mathrm{~mol} \% \mathrm{Bi}_{2} \mathrm{O}_{3}$ doping of the germanate glass was selected as the precursor glass for fabricating glass fibers and microspheres. The image of the Bi-doped glass fibers and microsphere is shown in Figs. 2(a) and 2(b), respectively. The diameters of the glass fibers range from 80 to $120 \mu \mathrm{m}$. The Bi-doped germanate glass microsphere is a solid sphere with a diameter of about $50 \mu \mathrm{m}$, a stem diameter of $5 \mu \mathrm{m}$, and a stem length of $100 \mu \mathrm{m}$ (as measured by using optical microscopy).
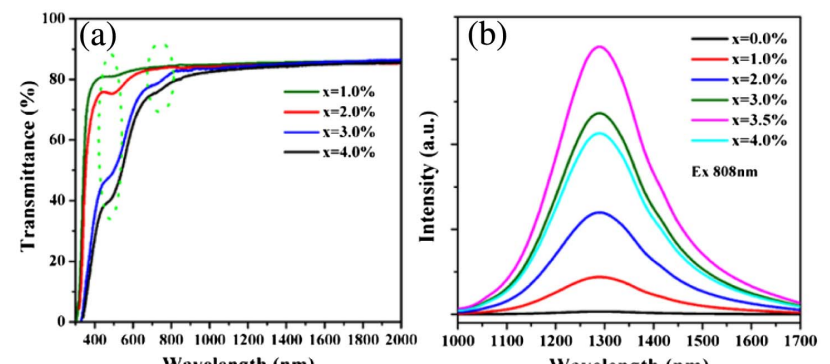

Fig. 1. (a) Transmission spectra of $x \mathrm{Bi}_{2} \mathrm{O}_{3}$-doped germanate glasses $(x=1.0,2.0,3.0$, and 4.0). The different absorption bands around 500 and $800 \mathrm{~nm}$ over the transmission spectra are marked by two green dashed elliptical circles. (b) Photoluminescence spectra of $x \mathrm{Bi}_{2} \mathrm{O}_{3}$-doped germanate glasses under $808 \mathrm{~nm}$ excitation $(x=0.0,1.0,2.0,3.0,3.5$, and 4.0). 

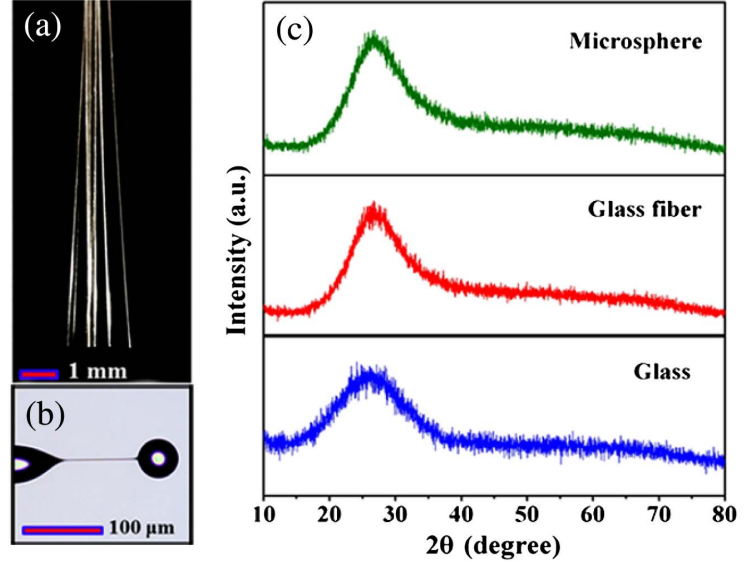

Fig. 2. (a) Microscope images of (a) Bi-doped germanate glass fiber and (b) microsphere. (c) XRD patterns of Bi-doped germanate glass, glass fibers and microspheres.

As previously reported by Fang et al. [34], Bi metals were easily precipitated during the rod-in-tube fiber drawing process. The transmittance and fluorescence properties of Bi-doped glass both deteriorated due to the precipitation of Bi metals, which prevents the lasing emission of Bi. For determining the amorphous state and crystalline phase in Bi-doped glasses, glass fibers, and microspheres, the XRD patterns were measured, as shown in Fig. 2(c). We see that the XRD patterns are all consistent with broad bands, attributed to the amorphous phase of the glass. There is no evidence of sharp diffraction peaks originating from the Bi metal or crystal in the observed XRD patterns. These results indicate that glass microspheres can be fabricated with relatively high optical quality due to (i) the rapid drawing speed that we used to make the glass fibers and (ii) the high temperature needed to make the microspheres. Therefore, the fabrication techniques that were used to obtain Bi-doped multicomponent glass microspheres avoid precipitation of the Bi metal, a fact that is important in order to obtain efficient Bi lasing in later experiments.

A schematic representation of the experimental apparatus used to achieve the Bi microsphere laser is shown in Fig. 3. Using a hydrogen-oxygen flame fiber pulling rig, a $1.0 \mu \mathrm{m}$ taper was fabricated by softening and stretching a section of $780 \mathrm{HP}$ silica optical fiber, similar to that described elsewhere [35], and this was used to couple the $808 \mathrm{~nm}$ pump source light, from a single mode, tunable external cavity laser, into the microsphere using evanescent field coupling. Relative positions of the

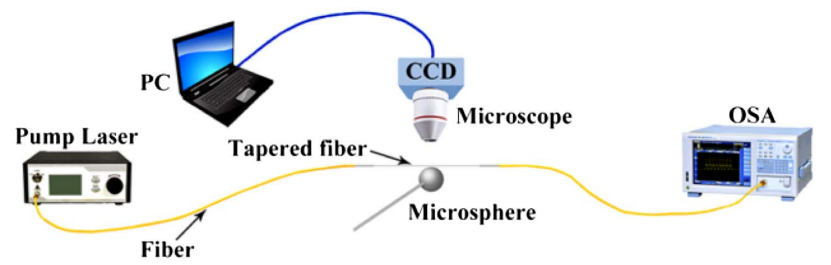

Fig. 3. Experimental setup for characterizing a Bi-doped microsphere laser. An $808 \mathrm{~nm}$ laser diode is used as the excitation source. taper and sphere were observed from two directions using two $20 \times$ microscope eyepieces attached to CCD cameras. The single-mode fiber used has the distinct advantage that it can allow efficient propagation of both excitation light and lasing emission. The fiber taper was placed above the Bi-doped germanate microsphere, which was held by the fiber stem and placed toward critical coupling with the tapered fiber. The output spectrum from the Bi-doped microsphere laser was monitored using an optical spectrum analyzer.

Figure 4(a) shows the WGMs obtained around $1310 \mathrm{~nm}$, as measured through the fiber taper coupler. Note that the pump power is below the threshold needed for lasing in the microcavity. The $Q$ factor was estimated to be about $2.5 \times 10^{5}$ using the wellknown formula $Q=\lambda /$ FWHM, where $\lambda$ and FWHM are the wavelength and FWHM of a single-mode resonance peak, respectively. Lasing from the Bi-doped microsphere was observed by adjusting the position of the microsphere and approaching it to the fiber taper. Figure 4(b) shows a typical lasing spectrum (as collected from the output pigtail of the fiber taper) recorded on the optical spectrum analyzer with a limited resolution of $0.05 \mathrm{~nm}$. The wavelength of the single-mode lasing peak is around $1305.8 \mathrm{~nm}$. This is the first experimental report for a Bi NIR laser based on a multicomponent glass. Lasing emission from the microsphere was observed by tuning the pump wavelength to a pump band resonance of the cavity and increasing the pump power to an approximate lasing threshold of $30 \mathrm{~mW}$. This threshold is overestimated because the absorbed pump power (circa $0.7 \%$ of the total input pump power of $30 \mathrm{~mW}$ ) was calculated without accounting for losses due to scattering. Future work should focus on refining the experimental setup to allow for more precise measurements of the loss mechanisms occurring in the microsphere-fiber taper system, especially surface scattering from the microsphere, as it appears to be by far the dominant loss mechanism for the pump. To obtain a high power transfer to the cavity from the fiber taper, light coupling into the microsphere should be improved. For this work, improving the coupling coefficient of the taper-sphere system relies on the phase-matching condition, i.e., simply obtaining size matching between the microsphere resonator and the fiber taper, as discussed in Refs. [36,37]. The polarization state of the pump source was adjusted using a polarization controller. However, the exact polarization state at the coupling fiber waist could not be
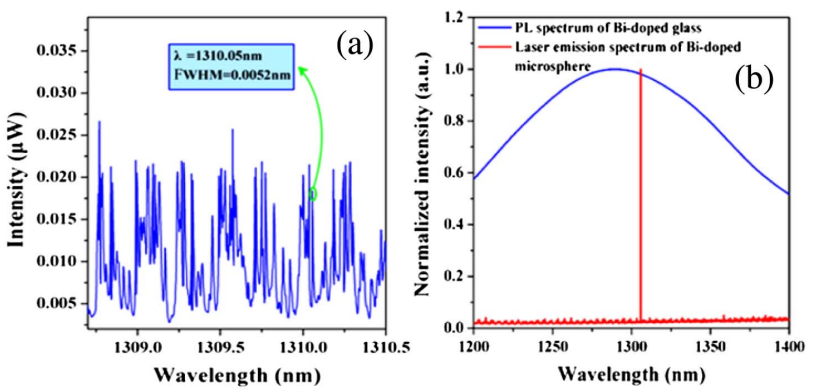

Fig. 4. (a) WGMs observed when light is coupled into doped microsphere via the fiber taper coupler. (b) Laser emission (red curve) from the Bi-doped microsphere when the absorbed pump power reaches $215 \mu \mathrm{W}$. As a reference, the fluorescence spectrum from the Bi-doped multi-component glass is also shown (blue curve). 


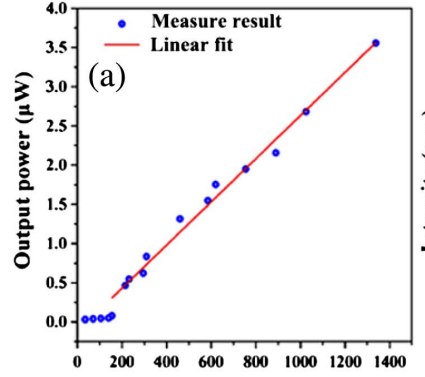

Absorbed pump power $(\mu \mathrm{W})$

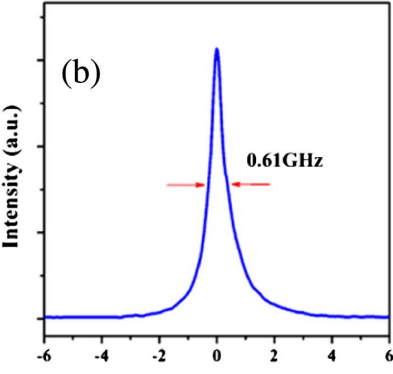

Frequency (GHz)

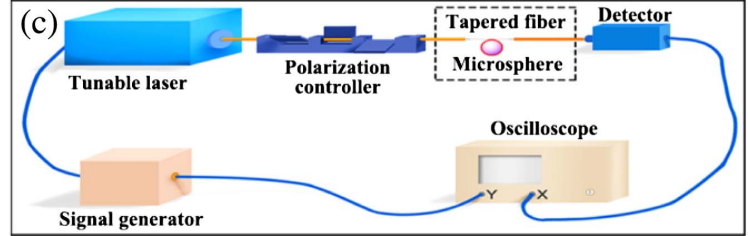

Fig. 5. (a) Microsphere laser output power as a function of estimated absorbed pump power at $1305.8 \mathrm{~nm}$. The straight red line is a linear fit to the experimental data. (b) Oscilloscope trace of the Bi-doped microsphere laser recorded as the pump is scanned in frequency. (c) Schematic of the experimental setup for the linewidth measurement of Bi laser emission.

determined with complete certainty due to the uncertain nature of light propagation in ultrathin fibers.

The output laser power collected from the fiber taper as a function of estimated total pump power absorbed is presented in Fig. 5(a). Below the pump threshold power at $215 \mu \mathrm{W}$, the emission of the microsphere presents as spontaneous radiation. The output power was observed to be as high as $3.56 \mu \mathrm{W}$ when the laser was operating in single-mode. In order to more precisely measure the linewidth of the single-mode laser emission, a pump laser launched from a frequency-scanned tunable laser was coupled into the microsphere via the fiber taper and a polarization controller. The frequency was repeatedly tuned over several $\mathrm{GHz}$ via piezo control, and the output signal was collected using a photodetector and viewed on an oscilloscope. The schematic of the experimental setup is shown in Fig. 5(c), and the trace of the Bi-doped microsphere laser output intensity recorded as the pump was scanned in frequency is presented in Fig. 5(b). From Fig. 5(b) it is clear that the linewidth is circa $0.61 \mathrm{GHz}(\sim 3.47 \mathrm{pm})$, which could be further improved by using a narrow linewidth pump laser and/or improving the quality of the gain microsphere. The output power of the Bi-doped microsphere laser is really low compared with that of a Bi-doped fiber laser presented so far $[15,16]$. However, the Bi-doped microsphere laser exhibits a lower pump threshold power and a narrower laser linewidth compared with the Bi-doped fiber laser. Furthermore, the compact physical size of this Bi-doped microsphere device makes it more suitable for an integrated optical system.

\section{CONCLUSION}

In conclusion, as a proof-of-concept, we have experimentally demonstrated a Bi-doped germanate glass microsphere laser with emissions around $1305.8 \mathrm{~nm}$ in a fiber tapered coupled system. Both the pump light and the lasing emission were guided through the fiber taper, providing an efficient coupling method to the microsphere cavity compared with what could be achieved using side-polished fiber or prism coupling methods. The estimated threshold pump power is $30 \mathrm{~mW}$, the threshold absorbed pump power is about $215 \mu \mathrm{W}$, and a measured output laser power as high as circa $3.56 \mu \mathrm{W}$ was observed with no sign of saturation. This Bi-doped multicomponent glass microsphere laser could be useful in a wide range of applications, in areas as diverse as NIR telecommunications, biomedical, or astrophysical applications, as an alternative to the more prevalent $1310 \mathrm{~nm}$ vertical cavity surface-emitting lasers [38].

Funding. National Natural Science Foundation of China (NSFC) (61575050); Key Program for International S\&T Cooperation Projects of China (2016YFE0126500); Key Program for Natural Science Foundation of Heilongjiang Province of China (ZD2016012); 111 Project (B13015); Fundamental Research Funds of the Central University; Harbin Engineering University (HEU).

Acknowledgment. This work was supported in part by the Okinawa Institute of Science and Technology Graduate University.

\section{REFERENCES}

1. J. Weber, J. Felten, B. Cho, and P. Nordine, "Glass fibres of pure and erbium-or neodymium-doped yttria-alumina compositions," Nature 393, 769-771 (1998)

2. S. Zhou, C. Li, G. Yang, G. Bi, B. Xu, Z. Hong, K. Miura, K. Hirao, and J. Qiu, "Self-limited nanocrystallization-mediated activation of semiconductor nanocrystal in an amorphous solid," Adv. Funct. Mater. 23, 5436-5443 (2013).

3. S. Jackson, "Towards high-power mid-infrared emission from a fibre laser," Nat. Photonics 6, 423-431 (2012).

4. F. Stutzki, C. Gaida, M. Gebhardt, F. Jansen, C. Jauregui, J. Limpert, and A. Tünnermann, "Tm-based fiber-laser system with more than 200 MW peak power," Opt. Lett. 40, 9-12 (2015).

5. Y. Tsang, B. Richards, D. Binks, J. Lousteau, and A. Jha, " $\mathrm{Tm}^{3+} / \mathrm{Ho}^{3+}$ codoped tellurite fiber laser," Opt. Lett. 33, 1282-1284 (2008).

6. Y. Fujimoto and M. Nakatsuka, "Optical amplification in bismuthdoped silica glass," Appl. Phys. Lett. 82, 3325-3326 (2003).

7. X. Meng, J. Qiu, M. Peng, D. Chen, Q. Zhao, X. Jiang, and C. Zhu, "Near infrared broadband emission of bismuth-doped aluminophosphate glass," Opt. Express 13, 1628-1634 (2005).

8. Y. Seo, Y. Fujimoto, and M. Nakatsuka, "Optical amplification in a bismuth-doped silica glass at $1300 \mathrm{~nm}$ telecommunications window," Opt. Commun. 266, 169-171 (2006).

9. Y. Arai, T. Suzuki, Y. Ohishi, S. Morimoto, and S. Khonthon, "Ultrabroadband near-infrared emission from a colorless bismuthdoped glass," Appl. Phys. Lett. 90, 261110 (2007).

10. V. Sokolov, V. Plotnichenko, and E. Dianov, "Origin of broadband near-infrared luminescence in bismuth-doped glasses," Opt. Lett. 33, 1488-1490 (2008).

11. S. Zhou, N. Jiang, B. Zhu, H. Yang, S. Ye, G. Lakshminarayana, J. Hao, and J. Qiu, "Multifunctional bismuth-doped nanoporous silica glass: from blue-green, orange, red, and white light sources to ultra-broadband infrared amplifiers," Adv. Funct. Mater. 18, 14071413 (2008).

12. V. Truong, L. Bigot, A. Lerouge, M. Douay, and I. Razdobreev, "Study of thermal stability and luminescence quenching properties of bismuth-doped silicate glasses for fiber laser applications," Appl. Phys. Lett. 92, 041908 (2008). 
13. Y. Fujimoto, "Bismuth doped silica glass and fiber," Rev. Laser Eng. 38, 869-875 (2010)

14. X. Guo, H. Li, L. Su, P. Yu, H. Zhao, J. Liu, and J. Xu, "Near-infrared broadband luminescence in $\mathrm{Bi}_{2} \mathrm{O}_{3}-\mathrm{GeO}_{2}$ binary glass system," Laser Phys. 21, 901-905 (2011).

15. E. Dianov, V. Dvoyrin, V. Mashinsky, A. Umnikov, M. Yashkov, and A. Gur'yanov, "CW bismuth fibre laser," Quantum Electron. 35, 10831084 (2005).

16. I. Razdobreev, L. Bigot, V. Pureur, A. Favre, G. Bouwmans, and M. Douay, "Efficient all-fiber bismuth-doped laser," Appl. Phys. Lett. 90, 031103 (2007).

17. I. Bufetov and E. Dianov, "Bi-doped fiber lasers," Laser Phys. Lett. 6, 487-504 (2009).

18. M. Kalita, S. Yoo, and J. Sahu, "Bismuth doped fiber laser and study of unsaturable loss and pump induced absorption in laser performance," Opt. Express 16, 21032-21038 (2008).

19. S. Norizan, W. Chong, S. Harun, and H. Ahmad, "O-band bismuthdoped fiber amplifier with double-pass configuration," IEEE Photon. Technol. Lett. 23, 1860-1862 (2011).

20. E. Dianov, "Bismuth-doped optical fibers: a challenging active medium for near-IR lasers and optical amplifiers," Light Sci. Appl. 1, e12 (2012).

21. T. Noronen, S. Firstov, E. Dianov, and O. Okhotnikov, "1700 nm dispersion managed mode-locked bismuth fiber laser," Sci. Rep. 6 , 24876 (2016).

22. I. Lobach, S. Kablukov, M. Skvortsov, E. Podivilov, M. Melkumov, S. Babin, and E. Dianov, "Narrowband random lasing in a bismuth-doped active fiber," Sci. Rep. 6, 30083 (2016).

23. M. Gorodetsky, A. Savchenkov, and V. Ilchenko, "Ultimate Q of optical microsphere resonators," Opt. Lett. 21, 453-455 (1996).

24. T. Kippenberg, S. Spillane, and K. Vahala, "Demonstration of ultrahigh-Q small mode volume toroid microcavities on a chip," Appl. Phys. Lett. 85, 6113-6115 (2004).

25. S. Spillane, T. Kippenberg, and K. Vahala, "Ultralow-threshold Raman laser using a spherical dielectric microcavity," Nature 415, 621-623 (2002).

26. Y. Wu, J. Ward, and S. Nic Chormaic, "Ultralow threshold green lasing

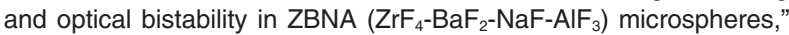
J. Appl. Phys. 107, 033103 (2010).
27. L. Tong, L. Hu, J. Zhang, J. Qu, Q. Yang, J. Lou, Y. Shen, J. He, and Z. Ye, "Photonic nanowires directly drawn from bulk glasses," Opt. Express 14, 82-87 (2006).

28. S. Zhou, H. Dong, H. Zeng, G. Feng, H. Yang, B. Zhu, and J. Qiu, "Broadband optical amplification in Bi-doped germanium silicate glass," Appl. Phys. Lett. 91, 061919 (2007).

29. Y. Zhao, M. Peng, A. Mermet, J. Zheng, and J. Quu, "Precise frequency shift of NIR luminescence from bismuth-doped $\mathrm{Ta}_{2} \mathrm{O}_{5}-\mathrm{GeO}_{2}$ glass via composition modulation," J. Mater. Chem. C 2, 78307835 (2014).

30. M. Peng, J. Qiu, D. Chen, X. Meng, and C. Zhu, "Broadband infrared luminescence from $\mathrm{Li}_{2} \mathrm{O}-\mathrm{Al}_{2} \mathrm{O}_{3}-\mathrm{ZnO}-\mathrm{SiO}_{2}$ glasses doped with $\mathrm{Bi}_{2} \mathrm{O}_{3}$," Opt. Express 13, 6892-6898 (2005).

31. N. Zhang, K. Sharafudeen, G. Dong, M. Peng, and J. Qiu, "Mixed network effect of broadband near-infrared emission in Bi-doped $\mathrm{B}_{2} \mathrm{O}_{3}-\mathrm{GeO}_{2}$ glasses," J. Am. Ceram. Soc. 95, 3842-3846 (2012).

32. M. Peng, J. Qiu, D. Chen, X. Meng, and C. Zhu, "Superbroadband $1310 \mathrm{~nm}$ emission from bismuth and tantalum codoped germanium oxide glasses," Opt. Lett. 30, 2433-2435 (2005).

33. J. Ren, L. Yang, J. Qiu, D. Chen, X. Jiang, and C. Zhu, "Effect of various alkaline-earth metal oxides on the broadband infrared luminescence from bismuth-doped silicate glasses," Solid State Commun. 140, 38-41 (2006).

34. Z. Fang, S. Zheng, W. Peng, H. Zhang, Z. Ma, G. Dong, S. Zhou, D. Chen, and J. Qiu, "Bismuth-doped multicomponent optical fiber fabricated by melt-in-tube method," J. Am. Ceram. Soc. 99, 856-859 (2016).

35. J. Ward, A. Maimaiti, V. Le, and S. Nic Chormaic, "Contributed review: optical micro-and nanofiber pulling rig," Rev. Sci. Instrum. 85, 111501 (2014).

36. A. Levi, R. Slusher, S. McCall, J. Glass, S. Pearton, and R. Logan, "Directional light coupling from microdisk lasers," Appl. Phys. Lett. 62, 561-563 (1993).

37. H. Hattori, Z. Li, D. Liu, I. Rukhlenko, and M. Premaratne, "Coupling of light from microdisk lasers into plasmonic nano-antennas," Opt. Express 17, 20878-20884 (2009).

38. V. lakovlev, G. Suruceanu, A. Caliman, A. Mereuta, A. Mircea, C. Berseth, A. Syrbu, A. Rudra, and E. Kapon, "High-performance single-mode VCSELs in the 1310-nm waveband," IEEE Photon. Technol. Lett. 17, 947-949 (2005). 